\title{
Recognizing the Payoff for Investing in Prevention
}

\author{
Susan R. Tortolero
}

Published online: 19 August 2011

(C) Springer Science+Business Media, LLC 2011

Early in August, we had a major victory for prevention. The U.S. Department of Health and Human Services released requirements for new health insurance plans to cover eight preventive women's health services at no cost for women (Institute of Medicine 2011). The recommended services are screenings for gestational diabetes, cervical cancer, HIV, and sexually transmitted infections; lactation counseling and breastfeeding support; screening and counseling for domestic violence; contraception; and yearly preventive care visits. These services were recommended by the Institute of Medicine Committee on Preventive Services for Women, who carefully considered the evidence upon which they based their recommendations. Major victories such as this one are the result of years of research from prevention scientists to understand modifiable risk factors; develop and test methods of screening and intervention; translate effective programs into practice; conduct effectiveness studies that also examine cost, dissemination, and adoption; and conduct

\section{S. R. Tortolero $(\square)$}

Health Promotion and Behavioral Sciences,

The University of Texas School of Public Health, Houston, TX, USA

e-mail: Susan.Tortolero@uth.tmc.edu

\section{S. R. Tortolero}

University of Texas Prevention Research Center,

Houston, TX, USA methodological studies to strengthen the ways in which we research these topics.

Manuscripts in this issue address important prevention topics such as drug use prevention in youth, the promotion of positive behaviors among young girls, an environmental intervention to increase access to fresh fruits and vegetables, the prevalence and emergence of cigar and cigarillo use among teens, suicide prevention, prevention of abuse, and ways Native American youth access health information. Studies in this issue reflect the prevention research continuum, from conducting needs assessments to more rigorous randomized designs. For example, the Rushing and Stephens article examines new technological avenues for reaching American Indian and Alaska Native youth, finding that as many as $75 \%$ of those youth reported searching online for health information. These data are already being used to design technology-based interventions for this high-risk population. At the other end of the research spectrum, Portwood and colleagues conducted a randomized trial to examine the impact of a primary prevention program, finding that the Adults and Children Together Against Violence Parents Raising Safe Kids program is a promising strategy to reduce child maltreatment in diverse communities. Another article by Cross and colleagues reminds us of the importance of training others to be agents of public health by examining approaches to better enhance and maintain suicide prevention gatekeeper skills over time. 
Investment in all types of prevention research is essential, and it is critically important that in these tough economic times, funding for prevention research is prioritized so we can continue to conduct these important studies and make gains in improving health and well-being. In addition, while funding to conduct studies is vital, funding to support public health infrastructure is also crucial.

The guest editorial in this issue describes a model for building such infrastructure to conduct prevention research. Drs. Ammerman, Harris, and Brownson and Mr. Tovar-Aguilar provide an overview of how the CDC Prevention Research Centers are advancing health by prioritizing health issues in the community, mobilizing teams to address these concerns, designing innovative solutions, training practitioners to deliver programs, and implementing the most feasible solutions to achieve high impact.

\section{References}

Institute of Medicine. (2011). Clinical preventive services for women: Closing the gaps. Washington, DC: The National Academies Press. 